\title{
CONCENTRAÇÃO DE ZN E MN NOS EFLUENTES DO BENEFICIAMENTO DE CARVÃO MINERAL E EM Typha domingensis PERS (TYPHACEAE) ${ }^{1}$
}

\author{
Jairo José Zocche², Micheli Freitas ${ }^{3}$ e Karin Esemann de Quadros ${ }^{4}$
}

\begin{abstract}
RESUMO - O estudo objetivou investigar a concentração de zinco e manganês nos efluentes do beneficiamento de carvão e em Typha domingensis Pers. Amostras de sedimento, água, raízes, caules e folhas ( $\mathrm{n}=8$ cada) foram coletadas no Lavador da Mina do Trevo, Siderópolis, SC, e em um banhado sem influência da mineração (área-controle). O conteúdo disponível dos metais no sedimento, dissolvido na água e total em T. domingensis, foi determinado por espectrofotometria de absorção atômica. Os dados de concentração de metais nos diferentes compartimentos e áreas foram submetidos ao teste "t", à análise de variância (ANOVA I) e à análise de correlação de Pearson (r). A significância estatística entre as diferenças verificadas na ANOVA foi avaliada por meio do teste SNK (Student-Newman-Keuls-Test), $\mathrm{P} \leq 0,05 \mathrm{e} \leq 0,01$. Zn e Mn apresentaram-se em concentrações mais elevadas no sedimento do que na água, nas duas áreas. Ambos os metais foram detectados na água em concentrações mais elevadas na área minerada do que na área-controle, enquanto no sedimento apenas o $\mathrm{Zn}$ seguiu essa tendência. $T$. domingensis concentrou $\mathrm{Zn}$ em valores mais elevados na raiz, enquanto o $\mathrm{Mn}$, nas folhas, evidenciando, ainda, concentrações mais elevadas de $\mathrm{Zn}$ na área minerada do que na área-controle. A explotação do carvão, portanto, disponibilizou Zn e Mn em concentrações mais elevadas na área minerada do que aquelas verificadas na área não minerada, comprometendo, assim, a biota. T. domingensis comportouse como espécie concentradora de metais, o que comprova sua potencialidade para utilização na fitorremediação de áreas degradadas.
\end{abstract}

Palavras-chave: Metais pesados, Banhados e Fitorremediação.

\section{ZINC AND MANGANESE CONTENTS IN EFFLUENTS FROM COAL TREATMENT AND Typha domingensis PERS (TYPHACEAE)}

\begin{abstract}
The objective of this study was to investigate the concentration of manganese and zinc in effluents from coal treatment and in Typha domingensis Pers. Samples of sediment, water, roots, stems and leaves ( $n=8$ each one) were collected in the Mina do Trevo Coal Washer, Siderópolis, SC, and in a swamp free of mining influence (control area). The contents of $\mathrm{Mn}$ and $\mathrm{Zn}$ in the sediment dissolved in the water and total in Typha domingensis Pers were determined by Atomic Absorption Spectrophotometry (AAS). Data on concentration of metals in the different compartments were submitted to t-test, one-way analysis of variance (ANOVA I) and Pearson correlation analysis ( $r$ ). The statistical significance between the differences found in the analysis of variance was evaluated by the Student-Newman-Keuls-Test, $P \leq 0.05$ and $\leq 0.01$. Zn and $M n$ presented higher concentrations in the sediment than in the water in both areas. Levels of Mn and $Z n$ in water showed to be higher in the mining area than in the control area, while in the sediment only $Z n$ followed this tendency. T. domingensis concentrated higher levels of Zn in the root and of Mn in the leaves, indicating higher $\mathrm{Zn}$ concentration in the mining area than in the control area. The coal exploitation has increased the available content of Zn and Mn in the mining area to concentrations higher than those recorded in the control area, causing thus damage to the biota. T. domingensis acted as an accumulator species of heavy metals, showing potential for use in phytoremediation of degraded areas.
\end{abstract}

Keywords: Heavy metals, Wetlands and Phytoremediation.

\footnotetext{
${ }^{1}$ Recebido em 20.11.2007 e aceito para publicação em 14.10.2009.

${ }^{2}$ Universidade do Extremo Sul Catarinense, Unidade Acadêmica Humanidades Ciências e Educação, Departamento de Ciências Biológicas, Criciúma, SC - Brasil. E-mail: <jjz@ unesc.net>.

${ }^{3}$ Universidade do Extremo Sul Catarinense - Criciúma, SC - Brasil. E-mail: <mfreitas@ unesc.net>.

${ }^{4}$ Fundação Universidade Regional de Blumenau, Centro de Ciência Exatas e Naturais, Departamento de Ciências Naturais, Blumenau, SC - Brasil. E-mail: <karin@ furb.br>.
} 


\section{INTRODUÇÃO}

A lavra mecanizada de carvão a céu aberto em Santa Catarina teve início na década de 1940 (CETEM, 2001) e desde então tem provocado alterações físicas, químicas e biológicas nos ecossistemas associados às áreas de mineração, comprometendo os recursos hídricos, o solo e a biota (COSTA et al., 2007).

A contaminação das bacias hidrográficas do Sul de Santa Catarina por efluentes da mineração do carvão é um problema real em nosso ambiente nos últimos anos (ZOCCHE, 2002). Observou-se que as áreas mineradas há muito tempo na Bacia Carbonífera Catarinense ainda contribuem para a geração de drenagens ácidas de mina (DAM), comprometendo os recursos hídricos das bacias hidrográficas que têm contato com elas (ZOCCHE, 2005).

Rejeitos recentes da mineração do carvão têm, usualmente, $\mathrm{pH}$ neutro ou levemente alcalino, que começa a decrescer quando a pirita se oxida (PROCHNOW e PORTO, 2000). O processo de oxidação da pirita contribui para abaixamento dos níveis de pH do solo e das águas, acelerando o intemperismo das argilas contidas nos estéreis e rejeitos da mineração, fazendo que a drenagem superficial contenha, além dos contaminantes típicos dos rejeitos da mineração, elevados teores de metais pesados (ZANARDI JR. e PORTO, 1991).

Mecanismos físico-químicos de retenção de metais em sedimentos incluem a imobilização via precipitação oxidativa, sedimentação de partículas, adsorção a sítios de trocas de cátions, complexação com matéria orgânica e redução a sulfatos (SKOUSEN et al., 1994). Sob condições de $\mathrm{pH}$ baixo há tendência à solubilização dos metais, enquanto sob condições de $\mathrm{pH}$ mais elevado ocorrem a absorção deles as partículas de argila e a complexação dos metais com a matéria orgânica, fatos observados por Zanardi Jr. e Porto (1991) ao compararem os parâmetros físico-químicos de um reservatório livre de contaminação por mineração com os das bacias de decantação de efluentes em áreas de mineração de carvão na Mina do Recreio em Butiá, RS.

A fração mais fina do sedimento tem importante papel na dinâmica dos metais pesados (BROOKS, 1983). Os ácidos húmicos, devido à sua relativa insolubilidade em meios ácidos, podem ser considerados como depósitos orgânicos de metais pesados (KABATA PENDIAS e PENDIAS, 1986). Assim, é de se supor que no compartimento onde haja maior teor de argila ou de matéria orgânica haja também maiores concentrações de metais pesados.

Recentemente, estudos relacionados a rejeitos sólidos e a efluentes da explotação do carvão, industriais e domésticos, têm sido desenvolvidos no sentido de: relacionar a presença de componentes tóxicos no substrato com a sucessão vegetal (ZOCCHE, 2005; COSTA et al., 2007); verificar o desenvolvimento de mecanismos de tolerância das espécies a metais pesados (AZOLLINI et al., 2006); verificar a possibilidade de utilização de efluentes na fertirrigação convencional de viveiros florestais (AUGUSTO et al., 2003); promover a fitorremediação e biopolimento de efluentes, utilizandose banhados construídos (wetlands) (MOTTAMARQUES et al., 2000; FREITAS et al., 2007; ZOCCHEDE-SOUZA et al., 2007).

Haddad et al. (2006) avaliaram a concentração de metais pesados (Zn, Cr e Ni) no sedimento de fundo, nas folhas e raízes de $T$. domingensis em um banhado construído em escala-piloto, para avaliar a eficiência do tratamento de efluentes de uma metalúrgica na Argentina.

Augusto et al. (2003) analisaram o aproveitamento da água residuária proveniente de um sistema de tratamento biológico de esgoto doméstico como alternativa à fertirrigação de viveiros florestais. Observaram bom desenvolvimento das mudas e ausência de deficiência nutritiva, de sintomas de fitotoxidez e de aumento na taxa de mortalidade das plantas em função da fertirrigação.

Vários estudos revelam que as plantas que crescem sobre áreas ricas em metais pesados apresentam adaptações fisiológicas, anatômicas e morfológicas em resposta ao estresse provocado pela ação desses metais. Soares et al. (2005) estudaram os efeitos de doses crescentes de $\mathrm{Cd}$ em solução nutritiva sobre o crescimento e absorção mineral de mudas de Eucalyptus maculata e E. urophylla e os respectivos sintomas de fitotoxidez em casa de vegetação. Observaram que, após uma semana de exposição aos tratamentos, as plantas exibiram pontuações avermelhadas nas nervuras, clorose internerval, necrose, murchamento das folhas e escurecimento das raízes, morte das gemas apicais e acentuada queda de folhas, ficando evidente a toxicidade de Cd às espécies de Eucalyptus estudadas e, a relação desta com a diminuição da translocação de $\mathrm{Cu}$ e $\mathrm{Fe}$ e com a diminuição dos teores foliares de $\mathrm{Mg}$. 
As respostas das plantas ao estresse químico da concentração de metais pesados podem, ainda, se manifestar na forma de produção de compostos orgânicos secundários como mecanismos de tolerância, conferindo à planta maior resistência ao estresse, pelo acúmulo desses metais, assim como propiciando alternativas para o desenvolvimento de tecnologias para o saneamento do ambiente, como a fitorremediação (ZOCCHE, 2002).

A habilidade das plantas para acumular metais as torna interessante objeto de pesquisa para testar e modelar teorias ecológicas sobre a evolução e sucessão vegetal, tanto quanto sobre a ciclagem de nutrientes e de metais. Várias plantas aquáticas são reconhecidas como acumuladoras de metais pesados em seus tecidos. As macrófitas aquáticas os absorvem, principalmente, pelas raízes, muito embora possa ser igualmente significante a absorção pelas folhas (VARDANYAN e INGOLE, 2006).

Apesar das condições extremas geradas nos ecossistemas aquáticos de áreas de mineração de carvão no Sul de Santa Catarina, observou-se que uma série de macrófitas aquáticas, como poáceas, ciperáceas, juncáceas, ninfeáceas e tifáceas, entre outras, se desenvolvem espontaneamente nesses ambientes. Essas plantas são o ponto de partida para o desenvolvimento de projetos de fitorremediação que visem à diminuição da geração de DAM e disseminação de compostos tóxicos nos ecossistemas aquáticos (ZOCCHE, 2005).

Diante do exposto, este estudo objetivou investigar a concentração de metais pesados ( $\mathrm{Zn}$ e $\mathrm{Mn}$ ) nos efluentes do beneficiamento de carvão e em Typha domingensis Pers., que cresce espontaneamente em uma bacia de decantação no Lavador da Mina do Trevo, Siderópolis, SC, visando avaliar o potencial da planta para a fitorremediação da drenagem ácida de mina.

\section{MATERIAL E MÉTODOS}

O estudo foi desenvolvido no ano de 2006, em uma bacia de decantação no Lavador da Mina do Trevo (6836125 N e 652500 E) e em um banhado natural de uma área não minerada (controle), na localidade de São Martinho Baixo (6834502 N e 649691 E), Siderópolis, SC, onde foram coletadas manualmente, em distâncias regulares de $10 \mathrm{~m}$, sobre uma transecção estabelecida a $5 \mathrm{~m}$ da margem em cada corpo d'água estudado, amostras ( $\mathrm{n}=8$ em cada área) de água, sedimento, raízes, caules e folhas de Typha domingensis Pers. (taboa). As amostras da planta foram obtidas de indivíduos adultos em período de crescimento vegetativo, sendo extraídas de cada planta coletada amostras de raiz, caule e folha.

T. domingensis é uma planta herbácea de 1,10 a 2,50 $\mathrm{m}$ de altura, com folhas que sobrepassam, igualam ou não alcançam a inflorescência; bainhas inferiores paulatinamente continuadas na lâmina e as superiores mais auriculadas; aurículas simétricas ou assimétricas; lâmina de 34 a $120 \mathrm{~cm}$ de comprimento por 0,5 a 1,6 cm de largura; anverso plano; e verso suavemente convexo. Floresce e frutifica de julho a fevereiro (REITZ, 1984).

As coletas de água subsuperficial e de sedimento foram mantidas sob refrigeração até o momento das análises. As amostras de raízes, caules e folhas de $T$. domingensis foram lavadas em água corrente, submetidas individualmente a banho ultrassom por $3 \mathrm{~min}$, lavadas novamente com água bidestilada, desidratadas em estufa a $100^{\circ} \mathrm{C}$ por $48 \mathrm{~h}$ e encaminhadas para análises. Na água, o conteúdo de metais pesados (Zn e Mn) foi analisado quanto à fração dissolvida seguindo APHA - Standart Methods (1998), enquanto no sedimento o conteúdo extraível foi determinado por meio de extração a frio com $\mathrm{HNO}_{3}(0,1 \mathrm{~N})$ e o total na planta, por meio de digestão total a quente com $\mathrm{HNO}_{3}$ suprapuro (KOTZ et al., 1972). As leituras foram efetuadas em espectrofotômetro de absorção atômica, acoplado a forno de grafite THGA Perkim-Elmer modelo SIMMA 6000.

Foram efetuadas em cada área estudada leituras do $\mathrm{pH}(\mathrm{n}=3)$ com phmêtro digital METRON 632. Na área minerada, além das leituras efetuadas no momento das coletas de água, foi analisada a série histórica de monitoramento do $\mathrm{pH}$ realizado pela empresa no ano de 2005, para se ter uma idéia do comportamento dessa variável ao longo do ano.

Os dados foram submetidos à estatística básica: média; desvio-padrão; análise de variância (ANOVAI). A significância estatística $(\mathrm{P} \leq 0,05$ e 0,01$)$ das diferenças observadas na concentração média dos metais na água, sedimento, raízes, caules e folhas, entre as áreas e entre os órgãos da planta em uma mesma área, foi testada por meio do teste " $t$ " e do SNK (Student-NewmanKeuls-Test). A correlação entre o conteúdo disponível dos metais na água e no sedimento e o total nas raízes, caules e folhas da planta, em cada respectiva área, foi verificada por meio do Coeficiente de Correlação de Pearson (r) (ZAR, 1985).

R. Árvore, Viçosa-MG, v.34, n.1, p.177-188, 2010 


\section{RESULTADOS E DISCUSSÃO}

O potencial hidrogeniônico $(\mathrm{pH})$ médio monitorado pela empresa, assim como o medido no momento das coletas de água na área minerada, ficou em torno de 5,2 unidades, enquanto na área-controle ficou em torno de 6,0 unidades. Em áreas de mineração de carvão os níveis do $\mathrm{pH}$ resultam da dissociação do ácido sulfúrico produzido a partir da oxidação da pirita $\left(\mathrm{FeS}_{2}\right)$ presente nos rejeitos (ZANARDI JR. e PORTO, 1991). Essas reações acidificam a água e aumentam a disponibilidade de vários elementos, o que compromete a biota; levam a seleção de um baixo número de espécies vegetais tolerantes, que passam a não encontrar competição por parte de outras espécies, gerando, assim, comunidades com baixa riqueza e elevado número de indivíduos por espécie, como foi observado na bacia de decantação estudada.

O pH é importante parâmetro que atua no controle da precipitação, mobilidade e biodisponibilidade dos íons metálicos. Quando em $\mathrm{pH}$ básico, a maior parte dos íons metálicos se precipita na forma de hidróxido ou de sais básicos (ESTEVES, 1988), enquanto em pH ácido uma série de elementos tem sua biodisponíbilidade aumentada (BROOKS, 1983).
Os conteúdos médios de Zn e Mn na água e nos sedimentos (Tabela 1) revelam que ambos os elementos se encontram em concentrações mais elevadas na área minerada do que na área-controle, evidenciando diferença estatística altamente significativa de Mn na água e Zn no sedimento e significativa de $\mathrm{Zn}$ na água, o que demonstra que a mineração do carvão está disponibilizando esses elementos para o meio.

Em relação às médias mundiais de concentração de metais pesados em águas continentais livres de contaminação, conforme Allen (1989), observou-se que, apesar dos tratamentos químicos efetuados pela empresa, ambos os elementos estão muito acima dos limites de normalidade (Tabela 1). De acordo com a Resolução CONAMA 357/2005 (BRASIL, 2005) que estabelece condições e padrões para o lançamento de efluentes, verificou-se que a concentração média do Mn (14,01 $\left.\mathrm{mg} . \mathrm{L}^{-1}\right)$ estava acima do valor máximo recomendado, que é de 1,0 mg.L -1 $^{-1}$ conteúdo dissolvido). Ye et al. (2001a), estudando wetlands contaminados por metais pesados provenientes da mineração de carvão nos EUA, constituídos de macrófitas aquáticas (Typha latifolia L. e Juncus effusius L.), constataram a presença de 6,4 $\mathrm{mg} \cdot \mathrm{L}^{-1}$ de $\mathrm{Mn}$ (conteúdo dissolvido) na água que fica entre os poros do sedimento.

Tabela 1 - Padrões de concentração e significância estatística da diferença de concentração média de Zn e Mn dissolvido na água $\left(\mathrm{mg} \cdot \mathrm{L}^{-1}\right)$ e trocável no sedimento $\left(\mu \mathrm{g} \cdot \mathrm{g}^{-1}\right)$, entre a área-controle e a área minerada $(\mathrm{n}=8$ para cada compartimento de cada área), determinada por meio do Student Test $(\mathrm{P} \leq 0,05$ e 0,01) .

Table 1 - Standards for concentration and statistical significance of the differences in the mean concentration of Zn and Mn dissolved in water $\left(\mathrm{mg}^{-1}\right)$ and exchangeable in the sediment $\left(\mu g . \mathrm{g}^{-1}\right)$ between control and mining areas $(n=8$ for each compartment of each area) determined by the Student Test $(P \leq 0.05$ and 0.01$)$.

\begin{tabular}{|c|c|c|c|c|}
\hline \multirow{3}{*}{$\begin{array}{l}\text { Parâmetros Estatísticos e } \\
\text { Padrões de Concentração }\end{array}$} & \multicolumn{4}{|c|}{ Conteúdo Dissolvido na Água } \\
\hline & \multicolumn{2}{|c|}{ Zinco $\left(\mathrm{mg} . \mathrm{L}^{-1}\right)$} & \multicolumn{2}{|c|}{ Manganês (mg.L $\mathrm{L}^{-1}$ ) } \\
\hline & Área-Controle & Área Minerada & Área-Controle & Área Minerada \\
\hline Média & $0,01 \mathrm{a}^{*}$ & $2,36 \mathrm{~b} *$ & $0,02 \mathrm{c} * *$ & $14,01 \mathrm{~d} * *$ \\
\hline Desvio-padrão & $1,24 \mathrm{E}-10$ & 2,12 & 0,06 & 3,60 \\
\hline Médias mundiais (água doce) ${ }^{1}$ & \multicolumn{2}{|c|}{$0,005-0,05$} & \multicolumn{2}{|c|}{$0,001-0,08$} \\
\hline Máximo permissível (água doce) ${ }^{2}$ & \multicolumn{2}{|c|}{0,18} & \multicolumn{2}{|c|}{0,1} \\
\hline Máximo permissível (efluentes) & \multicolumn{2}{|c|}{$5,0^{3}$} & \multicolumn{2}{|c|}{$1,0^{2}$} \\
\hline Parâmetros estatísticos e & \multicolumn{4}{|c|}{ Conteúdo Trocável no Sedimento } \\
\hline \multirow[t]{2}{*}{ Padrões de concentração } & \multicolumn{2}{|c|}{ Zinco $\left(\mu \mathrm{g} . \mathrm{g}^{-1}\right)$} & \multicolumn{2}{|c|}{ Manganês $\left(\mu \mathrm{g} . \mathrm{g}^{-1}\right)$} \\
\hline & Área-Controle & Área Minerada & Área-Controle & Área Minerada \\
\hline Média & $1,96 \mathrm{e}^{* *}$ & $11,41 \mathrm{f} * *$ & $19,01 \mathrm{~g}$ & $22,78 \mathrm{~g}$ \\
\hline Desvio-padrão & 1,83 & 4,05 & 6,73 & 7,88 \\
\hline Máximo permissível ${ }^{4}$ & \multicolumn{2}{|c|}{620} & \multicolumn{2}{|r|}{, } \\
\hline
\end{tabular}

${ }^{1}$ Conteúdo dissolvido, segundo Allen (1989); ${ }^{2 e 3}$ Conteúdos dissolvido e total, respectivamente, segundo Resolução CONAMA 357/2005; ${ }^{4}$ Conteúdo trocável, segundo Crommentuijn et al. (2000).

Médias seguidas por letras diferentes e por * nas linhas e por letras diferentes e ** indicam diferenças estatísticas significativas $(\mathrm{P} \leq 0,05)$ e diferenças estatísticas altamente significativas $(\mathrm{P} \leq 0,01)$, respectivamente.

R. Árvore, Viçosa-MG, v.34, n.1, p.177-188, 2010 
No que se refere ao compartimento dos sedimentos (Tabela 1), embora tenha sido detectada concentração média mais elevada de $\mathrm{Zn}$ na área minerada $\left(11,41 \mu \mathrm{g} . \mathrm{g}^{-1}\right)$ do que na área-controle $\left(1,96 \mu \mathrm{g} \cdot \mathrm{g}^{-1}\right)$, observou-se, em ambas as áreas, que tais valores estão bem abaixo dos limites considerados como normais, conforme Crommentuijn et al. (2000). Quanto ao conteúdo de Zn na água e Mn no sedimento, não foi possível estabelecer comparações, pois, no caso do Zn, foi determinado o conteúdo dissolvido, ao passo que a Resolução 357/2005 estabelece para esse elemento o conteúdo total. Já em relação ao Mn, não se encontraram, na literatura consultada, valores estabelecidos como permissíveis (aceitáveis ou normais).

Pompêo et al. (2004), ao estudarem a qualidade da água dos corpos contaminados pela mineração do carvão da bacia do rio Fiorita, em Siderópolis, $\mathrm{SC}$, encontraram valores de Mn total que variaram de 0,001 a $90 \mathrm{mg} \cdot \mathrm{L}^{-1}$. O conteúdo total de determinado elemento pouco informa sobre seu status de toxidez no meio, pois representa o valor potencial e não o valor real disponível para ser absorvido pelos organismos. A disponibilidade dependerá das variações nos parâmetros físicos e químicos, que poderão ou não gerar concentrações na forma trocável que virão a comprometer os processos biológicos. Por isso, é sempre preferível analisar a fração dissolvida ou trocável em vez do conteúdo total.

Machado et al. (2000) detectaram concentrações de Zn e Mn na forma extraível, nos sedimentos de cursos d'água da Bacia Carbonífera do Rio Grande do Sul, que variaram de 0,81 a $9,78 \mu \mathrm{g} \cdot \mathrm{g}^{-1}$ e de 5,23 a $110,72 \mu \mathrm{g} . \mathrm{g}^{-1}$, respectivamente. Se comparar com os valores detectados neste estudo (Tabela 1 ), verifica-se que nos sedimentos da bacia de decantação foram evidenciados valores de $\mathrm{Zn}$ superiores a estes, na ordem de duas vezes, e de Mn inferiores, na ordem de três vezes. Essas variações podem ser explicadas pelo fato de que, nas áreas estudadas no RS, as coletas foram feitas em vários pontos, em drenagens naturais, e neste estudo, na bacia de decantação dos efluentes do lavador. Tais fatos influenciam a geoquímica dos sedimentos, uma vez que as drenagens naturais recebem aporte de materiais diversos, além de conterem uma biota diversificada, o que determina a sua dinâmica, que é diferente daquela observada em uma bacia de decantação.

A flutuação do volume de água é outro fator que pode influir nos níveis de concentração de metais pesados de um corpo receptor. Além da entrada de efluentes, a elevação do nível da água da bacia de decantação do Lavador da Mina do Trevo se dá pelo aporte subsuperficial, que percola pelas pilhas de rejeitos e estéreis, levando, principalmente em épocas de chuvas intensas, ao extravasamento temporário, o que põe a "água" em contato com materiais em diferentes estados de intemperização, carreando para dentro da bacia de decantação, quantidades de metais pesados maiores do que aquelas contidas nos efluentes.

Foram observados, tanto na área-controle quanto na área minerada (Tabela 2), valores médios de concentração de $\mathrm{Zn}$ e Mn mais elevados nos sedimentos do que na água, evidenciando-se diferença estatisticamente significativa em ambos os elementos, corroborando a tendência observada por Duó et al. (2006). Esse fato pode ser explicado pela presença de maior quantidade de partículas de argila sedimentadas do que em suspensão na água.

Tabela 2 - Significância estatística da diferença de concentração média de Zn e Mn, entre o sedimento $\left(\mu g . g^{-1}\right)$ e a água $\left(\mathrm{mg} . \mathrm{L}^{-1}\right)$ em cada respectiva área estudada $(\mathrm{n}=8$ para cada compartimento de cada área), determinada por meio do Student Test $(\mathrm{P} \leq 0,05)$.

Table 2 - Statistical significance of the difference in the mean concentration of $Z n$ and Mn between sediment $\left(\mu g . g^{-1}\right)$ and water $\left(m g . L^{-1}\right)$ in each respective studied area $(n=8$ for each compartment of each area) determined by the Student Test $(P \leq 0.05)$.

\begin{tabular}{lccc}
\hline Áreas de Estudo & \multicolumn{2}{c}{ Concentração Média Zinco } & \multicolumn{2}{c}{ Concentração Média Manganês } \\
\cline { 2 - 4 } & \multicolumn{1}{c}{ Sedimento $\mu \mathrm{g} \cdot \mathrm{g}^{-1}$} & Água mg.L ${ }^{-1}$ & ${\text { Sedimento } \mu \mathrm{g} \cdot \mathrm{g}^{-1}}^{\text {Água mg.L }^{-1}}$ \\
\hline Não minerada & $1,96 \mathrm{a}$ & $0,01 \mathrm{~b}$ & $19,00 \mathrm{c}$ \\
Minerada & $11,41 \mathrm{e}$ & $2,36 \mathrm{f}$ & $22,78 \mathrm{~g}$ \\
\hline
\end{tabular}

Letras diferentes nas linhas indicam diferença estatística significativa entre as concentrações médias na água e no sedimento em uma mesma área $(\mathrm{P} \leq 0,05)$. 
A sorção é o destino dominante do Zn em ambientes aquáticos, sobretudo em condições de $\mathrm{pH}$ abaixo de 7 , sendo prontamente transportado para águas superficiais devido à sua mobilidade (SMITH et al., 1995). Prendese predominantemente ao material suspenso antes de ser acumulado no sedimento, no entanto a ressolubilização em fase aquosa é possível sob certas condições físicoquímicas, como na presença de ânions solúveis, na ausência de matéria orgânica, minerais de argila e hidróxidos de ferro e manganês, baixo pHe salinidade elevada (SOARES et al., 2001). A disponibilidade do Mn por sua vez, tende a ser aumentada em meios medianamente ácidos e, assim como a do Zn, pode ser muito baixa em meios fortemente ácidos (ALLEN, 1989).

Os conteúdos totais dos metais em T. domingensis (Tabela 3) mostram que o $\mathrm{Zn}$ encontra-se em concentrações mais elevadas na área minerada do que na controle na ordem de 18, 31,5 e 6,5 vezes nas raízes, nos caules e nas folhas, respectivamente, evidenciando diferença estatística altamente significativa (raízes e caules) e significativa (folhas), enquanto o conteúdo médio de Mn nos caules e nas folhas não diferiu estatisticamente entre as áreas, diferindo significativamente nas raízes, onde foi detectado na ordem de 1,6 vez mais baixo na área minerada. Emrelação às médias mundiais, conforme Allen (1989), observou-se que apenas as concentrações médias de Zn nas raízes, na área minerada estavam acima dos limites de normalidade.

Foi observado que o elemento $\mathrm{Zn}$ nas folhas de $T$. domingensis segue o mesmo padrão verificado na água e no sedimento na bacia de decantação de efluentes da mineração (Tabela 1), isto é, os valores médios detectados estão mais elevados nesta do que na área-controle. Isso significa dizer que as atividades de mineração do carvão estão disponibilizando $\mathrm{Zn}$ para o meio, e a planta, por sua vez, está absorvendo e concentrando-os em valores mais elevados, principalmente nas raízes e folhas.

Tabela 3 - Padrões de concentração e significância estatística da diferença de concentração média do conteúdo total de $\mathrm{Zn}$ e $\mathrm{Mn}\left(\mu \mathrm{g} . \mathrm{g}^{-1}\right)$ nas raízes, caules e folhas de Typha domingensis Pers., entre a área-controle e a área minerada ( $\mathrm{n}=8$ para cada órgão em cada área), determinada por meio do Student Test $(\mathrm{P} \leq 0,05$ e 0,01$)$.

Table 3 - Standards for concentration and statistical significance of the difference in the mean concentration of total content of $\mathrm{Zn}$ and $\mathrm{Mn}\left(\mu \mathrm{g} . \mathrm{g}^{-1}\right)$ in the roots, stems and leaves of Typha domingensis Pers. between the control and mining areas $(n=8$ for each vegetable organ in each area) determined by the Student Test $(P \leq 0.05$ and 0.01 ).

\begin{tabular}{|c|c|c|c|c|}
\hline \multirow{3}{*}{$\begin{array}{l}\text { Parâmetros Estatísticos } \\
\text { Padrões de Concentração }\end{array}$} & \multicolumn{4}{|c|}{ Conteúdo Total nas Raízes } \\
\hline & \multicolumn{2}{|c|}{ Zinco $\mu \mathrm{g} \cdot \mathrm{g}^{-1}$} & \multicolumn{2}{|c|}{ Manganês $\mu \mathrm{g} \cdot \mathrm{g}^{-1}$} \\
\hline & Área-Controle & Área Minerada & Área-Controle & Área Minerada \\
\hline Média & 13,72 a $* *$ & $247,50 \mathrm{~b} * *$ & $262,87 \mathrm{c} *$ & $161,50 \mathrm{~d} *$ \\
\hline Desvio-padrão & 2,82 & 146,06 & 106,01 & 35,46 \\
\hline Médias mundiais ${ }^{a}$ & \multicolumn{2}{|c|}{$15-100$} & \multicolumn{2}{|c|}{$50-1000$} \\
\hline \multirow[t]{3}{*}{ Parâmetros estatísticos } & \multicolumn{4}{|c|}{ Conteúdo Total nos Caules } \\
\hline & \multicolumn{2}{|c|}{ Zinco } & \multicolumn{2}{|c|}{ Manganês } \\
\hline & Área-Controle & Área Minerada & Área-Controle & Área Minerada \\
\hline Média & 1,39 e $* *$ & $43,85 \mathrm{f} * *$ & $200,46 \mathrm{~g}$ & $212,5 \mathrm{~g}$ \\
\hline Desvio-padrão & 2,77 & 25,80 & 94,96 & 46,80 \\
\hline Médias Mundiais a & \multicolumn{2}{|c|}{$5-100$} & \multicolumn{2}{|c|}{$50-1000$} \\
\hline Parâmetros Estatísticos e & \multicolumn{4}{|c|}{ Conteúdo Total nas Folhas } \\
\hline \multirow[t]{2}{*}{ Padrões de Concentração } & \multicolumn{2}{|c|}{ Zinco } & Manganês & \\
\hline & Área-Controle & Área Minerada & Área-Controle & Área Minerada \\
\hline Média & $7,87 \mathrm{~h} *$ & $52,36 \mathrm{i} *$ & $803,75 \mathrm{j}$ & $815,75 \mathrm{j}$ \\
\hline Desvio-padrão & 2,28 & 36,09 & 474,44 & 272,63 \\
\hline Médias mundiais ${ }^{1}$ & \multicolumn{2}{|c|}{$15-100$} & \multicolumn{2}{|c|}{$50-1000$} \\
\hline
\end{tabular}

${ }^{1}$ Segundo Allen (1989).

Médias seguidas por letras diferentes e por * nas linhas e por letras diferentes $\mathrm{e}^{* *}$ indicam diferenças estatísticas significativas $(\mathrm{P} \leq 0,05)$ e diferenças estatísticas altamente significativas $(\mathrm{P} \leq 0,01)$, respectivamente. * Significativo $(\mathrm{P} \leq 0,05) \mathrm{e}$ $* *$ altamente significativo $(\mathrm{P} \leq 0,01)$.

R. Árvore, Viçosa-MG, v.34, n.1, p.177-188, 2010 
Esse fato, ao contrário de ser ruim, oferece boas perspectivas de utilização da espécie em trabalhos de fitorremediação em áreas degradadas, por meio do biopolimento de efluentes tratados por processos físicoquímicos convencionais, em função da capacidade de bioacumulação desse elemento em seus tecidos foliares. Hadad et al. (2006) detectaram variação na concentração média de $\mathrm{Zn}$ nas folhas de $T$. domingensis de $6,1 \mu \mathrm{g} \cdot \mathrm{g}^{-1}$ a 39,0 $\mu \mathrm{g} \cdot \mathrm{g}^{-1}$, entre o início e o final do período de exposição aos efluentes (425 dias). Concluíram que T. domingensis, entre outras espécies, alcançou a taxa de produção de biomassa mais elevada em ambientes alterados por efluentes ácidos do que em ambientes naturais sem alteração antrópica. Afirmaram que essa espécie é altamente eficiente para o tratamento de efluentes enriquecidos com metais pesados e baixo $\mathrm{pH}$.

Ye et al. (2001b) verificaram que a concentração de Fe, S, Cd, B e Zn foi mais elevada nos tecidos das raízes e do caule do que dos brotos e folhas de Typha latifolia, em alguns casos, inclusive, maiores do que 60 vezes, ocorrendo o inverso em Mn, que mostrou concentrações mais elevadas nas raízes e nos caules. Detectaram concentrações de $\mathrm{Zn}$ iguais a 27 e $42 \mu \mathrm{g} . \mathrm{g}^{-1}$ nos brotos e raízes, respectivamente, enquanto as de Mn foram iguais a $1.097 \mathrm{e} 200 \mu \mathrm{g} . \mathrm{g}^{-1}$ respectivamente. Observaram, ainda, que T. latifolia acumulou manganês em maiores concentrações nas folhas e brotos do que nos rizomas e raízes, resultado também obtido por Taylor e Crowder (1983) e Fernandes e Henriques (1990).

As espécies vegetais, de modo geral, apresentam grande variação quanto à absorção de metais pesados (HART et al., 1998). As raízes, geralmente, constituem o principal órgão envolvido na absorção e, portanto, quase sempre as maiores concentrações de metais pesados são, também, encontradas nessa parte da planta (GRANT et al., 1998). Em plantas aquáticas, entretanto, as folhas estabelecem contato direto com a solução que contém o metal pesado e, portanto, teoricamente absorvem tanto quanto as raízes (TAYLOR e CROWDER, 1983).

Plantas aquáticas, pela própria condição do meio, absorvem elementos pela raiz, pelo caule e pelas folhas, dependendo muito do contato que cada órgão tem com a água ou com o sedimento. A absorção de metais pesados por folhas é pouco conhecida e, menos ainda, a influência que isso teria sobre a absorção pelas raízes e a subsequente translocação para a parte aérea (CAKMAK et al., 2000).

T. domingensis evidenciou acumulações de $\mathrm{Zn}$ mais elevadas em ambas às áreas na seguinte ordem: raízes > folhas > caules; enquanto de Mn: folhas > caules $>$ raízes (área minerada) e folha $>$ raiz $>$ caule (controle). Na área-controle foi observada diferença estatística significativa na concentração média de Zn entre os três órgãos, enquanto na área minerada apenas a concentração média nas raízes diferiu estatisticamente das concentrações dos caules e das folhas, cujos valores detectados nesses dois últimos órgãos não evidenciaram diferença estatística significativa entre si. Em relação ao Mn, foi observado que tanto na área minerada quanto na área-controle apenas a concentração média das folhas evidenciou diferença estatística significativa em relação aos outros dois órgãos (Tabela 4).

Zocche (2005), ao analisar a concentração de Fe, Mn e Zn em Brachiaria decumbens em áreas de depósitos de cinza de carvão mineral, verificou concentrações mais elevadas desses elementos nas raízes do que nas folhas. $\mathrm{O}$ fato de se encontrar maiores concentrações de metais nas raízes demonstra que estes são imobilizados, na maior parte, nos locais de absorção,

Tabela 4-Significância estatística da diferença de concentração média de metais $\left(\mu \mathrm{g} . \mathrm{g}^{-1}\right)$ entre as raízes, caules e folhas de Typha domingensis Pers., em cada respectiva área $(\mathrm{n}=8$ para cada órgão em cada área), determinada por meio da análise de variância - ANOVA I e testada por meio do SNK $(\mathrm{P} \leq 0,01)$.

Table 4 - Statistical significance of the difference in the mean concentration of metals $\left(\mu g^{-1} g^{-1}\right)$ between the roots, stems and leaves of Typha domingensis Pers. in each respective area $(n=8$ for each vegetable organ in each area) determined by the one-way analysis of variance - ANOVA I and tested by the $S N K(P \leq 0.01)$.

\begin{tabular}{|c|c|c|c|c|c|c|}
\hline \multirow[t]{2}{*}{ Áreas de Estudo } & \multicolumn{3}{|c|}{ Concentração Média Zinco } & \multicolumn{3}{|c|}{ Concentração Média Manganês } \\
\hline & Raiz $\mu g . g^{-1}$ & Caule $\mu \mathrm{g} \cdot \mathrm{g}^{-1}$ & Folha $\mu \mathrm{g} \cdot \mathrm{g}^{-1}$ & Raiz $\mu g . g^{-1}$ & Caule $\mu \mathrm{g} \cdot \mathrm{g}^{-1}$ & Folha $\mu \mathrm{g} . \mathrm{g}^{-1}$ \\
\hline Controle & $13,72 \mathrm{a}$ & $1,39 \mathrm{~b}$ & $7,87 \mathrm{c}$ & $262,87 \mathrm{~d}$ & $200,46 \mathrm{~d}$ & 803,75 e \\
\hline Minerada & $247,50 \mathrm{f}$ & $43,85 \mathrm{~g}$ & $52,36 \mathrm{~g}$ & $161,50 \mathrm{~h}$ & $212,50 \mathrm{~h}$ & $815,75 \mathrm{i}$ \\
\hline
\end{tabular}

Letras iguais nas linhas indicam que não há diferença estatística significativa entre as médias (P $\leq 0,01)$.

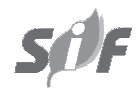

R. Árvore, Viçosa-MG, v.34, n.1, p.177-188, 2010 
provocando menores danos nas partes superiores das plantas estressadas (QUARITI et al., 1997). A imobilização de metais-traço nas raízes das plantas é um fenômeno comum, especialmente quando os suprimentos de micro e macronutrientes são suficientes (KABATA-PENDIAS e PENDIAS, 1986).

Estudos sobre fitotoxicidade dos metais pesados, assim como da tolerância das espécies aos estresses impostos pelo excesso destes, são essenciais para a seleção de plantas tolerantes e ao desenvolvimento da fitorremediação. Estudos bioquímicos têm demonstrado que a quelação dos metais pesados tem assegurado a tolerância das plantas aos excedentes de íons tóxicos no substrato. Os efeitos tóxicos dos metais, mesmo translocados pelo xilema, podem ser minimizados pela quelação, o que pode ocorrer ainda na raiz. A desintoxicação ou imobilização dos metais pode ser desencadeada pelas células sintetizadoras de ácidos orgânicos polipeptídeos que quelam os metais pesados (CUMMING e TAYLOR, 1990).

As raízes, geralmente, constituem o principal órgão das plantas terrestres envolvido na absorção e, portanto, quase sempre as maiores concentrações de metais pesados são, também, encontradas nessa parte da planta (GRANT et al., 1998). Em plantas aquáticas, entretanto, pelo fato de folhas também estabelecem contato direto com a solução de absorção que contém o metal pesado, elas podem absorver tanto quanto as raízes (TAYLOR e CROWDER, 1983; CAKMAK et al., 2000).

Via de regra, concentrações elevadas de metais pesados no meio implicam concentrações elevadas nas plantas, no entanto, como a absorção e concentração é espécie específica e varia de órgão para órgão, diferentes plantas podem acumular diversas concentrações de metais (ZOCCHE, 2002). A análise de correlação entre a concentração dos metais estudados no sedimento e na água e a concentração desses nos tecidos de $T$. domingensis (Tabela 5) revelou correlação positiva significativa apenas entre a concentração de $\mathrm{Zn}$ na água e nas raízes $\left(\mathrm{r}_{0,05 ; 6}=0,80\right)$ e na água e nos caules $\left(\mathrm{r}_{0,05 ; 6}=0,84\right)$ e altamente significativa entre a concentração na água e nas folhas $\left(\mathrm{r}_{0,001 ; 6}=0,97\right)$.

$\mathrm{Na}$ forma dissolvida na água, os elementos encontram-se diretamente disponíveis para serem absorvidos pelas plantas, enquanto nos sedimentos, mesmo estando disponíveis, podem estar formando ligações fracas com componentes orgânicos, argilas, ou estar adsorvidos a diversas superfícies. Isso pode explicar, em parte, o fato de que, embora os valores de concentração de $\mathrm{Zn}$ tenham sido mais elevados no sedimento do que na água, o coeficiente de correlação entre a concentração desse elemento no sedimento e nos órgãos de $T$. domingensis não evidenciou significância estatística. O padrão de concentração, observado neste estudo, em ambos os metais em T. domingensis comprovam o comportamento das plantas em concentrar elementos de forma diferenciada em diferentes órgãos. Esse fato é muito importante quando se pretende utilizar uma espécie para a fitoextração de elementos tóxicos dos efluentes, pois permite a retirada destes, por meio de colheitas sucessivas das partes aéreas das plantas (FREITAS et al., 2007).

Tabela 5 - Correlação entre a concentração média de Zn e Mn no sedimento $\left(\mu \mathrm{g} \cdot \mathrm{g}^{-1}\right)$ na água $\left(\mathrm{mg} . \mathrm{L}^{-1}\right)$ e o respectivo conteúdo $\left(\mu \mathrm{g} . \mathrm{g}^{-1}\right)$ nas raízes, caules e folhas de Typha domingensis Pers., em cada respectiva área estudada $(\mathrm{n}=8$ para cada compartimento de cada área), determinada pelo Coeficiente de Correlação de Pearson (r).

Table 5 - Correlation between the mean concentration of $\mathrm{Zn}$ and $\mathrm{Mn}$ in the sediment $\left(\mu g . g^{-1}\right)$, water $\left(m g . L^{-1}\right)$ and the respective content $\left(\mu g . g^{-1}\right)$ in the roots, stems and leaves of Typha domingensis Pers. in each respective studied area $(n=8$ for each compartment of each area) determined by the Pearson's correlation coefficient $(r)$.

\begin{tabular}{lccc}
\hline Compartimentos & \multicolumn{2}{c}{ Área-Controle } & \multicolumn{2}{c}{ Área Minerada } & Zinco \\
\cline { 2 - 4 } & Zinco & Manganês & 0,24 \\
\hline Sedimento x Raiz & $-0,03$ & 0,43 & 0,18 \\
Sedimento x Caule & $-0,22$ & 0,36 & 0,05 \\
Sedimento x Folha & $-0,13$ & 0,33 & $-0,34$ \\
Água x Raiz & 0,00 & 0,27 & $0,80 *$ \\
Água x Caule & 0,00 & 0,41 & 0,44 \\
Água x Folha & 0,00 & 0,25 & 0,20 \\
\hline
\end{tabular}

* Significativo $\mathrm{P} \leq 0,05$ e ** altamente significativo $\mathrm{P} \leq 0,01$.

R. Árvore, Viçosa-MG, v.34, n.1, p.177-188, 2010 


\section{CONCLUSÕES}

A mineração e o beneficiamento do carvão disponibilizaram quantidades de zinco e manganês na forma biodisponível em concentrações mais elevadas do que o padrão verificado nas áreas não mineradas do entorno da área estudada, o que compromete o estabelecimento e desenvolvimento da biota em áreas mineradas e não recuperadas.

Typha domingensis concentrou $\mathrm{Zn}$ na raiz e Mn nas folhas, comportando-se como espécie concentradora de metais, o que comprova sua potencialidade para a utilização na fitorremediação de drenagens ácidas de mina.

\section{REFERÊNCIAS}

ALLEN, S. E. Chemical analysis of ecological materials. 2.ed. London: Blackwell Scientific Publications, 1989. 368p.

AMERICAN PUBLIC HEALTH ASSOCIATION APHA. Standart methods for examination of water and wasterwader. 20.ed. Washington: AWWA, 1998. 1530p.

AZZOLINI, M. et al. Identificando os efeitos de $\mathrm{Cd}$ em gemas apicais de Ricinus communis $\mathrm{L}$ (Euphorbiaceae). In: CONGRESSO NACIONAL DE BOTÂNICA, 57., 2006, Gramado. Anais ... Gramado: Universidade Federal do Rio Grande do Sul, 2006. p.115-117.

AUGUSTO, D. C. C. et al. Utilização de esgotos domésticos tratados através de um sistema biológico na produção de mudas de croton floribundus spreng. (capixingui) e copaifera langsdorffii desf. (copaíba). Revista Árvore, v.27, n.3, p.335-342, 2003.

BRASIL. Resolução CONAMA 357/2005. Dispõe sobre a classificação dos corpos de água e diretrizes ambientais para seu enquadramento, bem como estabelece as condições e padrões de lançamento de efluentes e dá outras providências. Diário Oficial da República Federativa do Brasil. Brasília. Disponível em: <http://www.mma.gov.br/port/ conama $>$ Acesso em: 13 de maio de 2007.

BROOKS, R. R. Biological methods of prospecting for minerals. New York: Wiley-Intersciense, 1983. 322p.
CAKMAK, I. et al. Uptake and retranslocation of leaf-applied cadmium $\left({ }^{109} \mathrm{Cd}\right)$ in diploid, tetraploid and hexaploid wheats. Journal of

Experimental Botany, v.51, n.343, p.221226, 2000.

CENTRO DE TECNOLOGIA MINERAL - CETEM. Projeto conceitual para recuperação ambiental da Bacia Carbonífera Sul Catarinense. Relatório Técnico elaborado para o SIECESC, 2001. 2v.

COSTA, S.; ZOCCHE, J. J.; ZOCCHE-DE-SOUZA, $\mathrm{P}$. Absorção de metais pesados $(\mathrm{Zn}$ e $\mathrm{Pb})$ por Axonopus obtusifolius (Raddi) em áreas degradadas pela mineração de carvão, SC, Brasil. Revista Brasileira de Biociências, v.5, Suplemento 1, p.795-797, 2007.

CROMMENTUIJN, T. et al. Maximum permissible and negligible concentrations for metals and metalloids in the Netherlands, taking into account background concentrations. Journal of Environmental Management, v.60, n.2, p.121-143, 2000.

CUMMING, J. R.; TAYLOR, G. Mechanisms of metal tolerance in plants: physiological adaptations for exclusion of metal ions from the cytoplasm. In: ALSCHER, R. G.; CUMMING, J. R. Stress responses in plants: adaptation and acclimation mechanisms. New York: Wiley-Liss, 1990. p.329-356.

DUÓ, D. A. et al. Determinação de metais pesados em amostras de água e sedimento do córrego dos Bagres, no município de Franca, São Paulo. O Biólogo, v. 68, Suplemento 2, p.326-329, 2006.

ESTEVES, F. A. Fundamentos de limnologia. Rio de Janeiro: Interciência/FINEP, 1988. 575p.

FERNANDES, J. C.; HENRIQUES, F. S. Metal levels in soils and cattail (Typha latifolia L.) plants in a pyrite mine area at Lousal. International Journal of Environmental Studies, v.36, n.3, p.205-210, 1990.

FREITAS, M.; ZOCCHE, J. J.; QUADROS, K.E. Metais pesados (Mn e Zn) em Typha domingensis Pers. em áreas de mineração de carvão. Revista Brasileira de Biociências, v.5, Suplemento 1, p.789-791, 2007.

R. Árvore, Viçosa-MG, v.34, n.1, p.177-188, 2010 
GRANT, C. A. et al. Cadmium accumulation in crops. Canadian Journal of Plant Science, v.78, n.1, p.1-17, 1998.

HADAD, H. R.; MAINE, M. A.; BONETTO, C. A. Macrophyte growth in a pilot-scale constructed wetland for industrial wastewater treatment. Chemosphere, v. 63, n.10, p.17431753, 2006.

HART, J. J. et al. Characterization of cadmium binding, uptake, and translocation in intact seedlings of bread and durum wheat cultivars. Plant Physiology, v.116, n.4, p.1413-1420, 1998.

KABATA-PENDIAS, A.; PENDIAS, H. Trace elements in soil plants. Boca Raton: CRC Press, 1986. 315p.

KOTZ, L. et al. Aufschluß biologister matrices für die bestmmung sehr niedriger spurenelementgehalte bei gegrenster einwaage mit salpetesäure unter druck in einem teflongefaß. Fresenius' Journal of Analytical Chemistry, v.260, n.3, p.207209, 1972.

MACHADO, N. A. F.; RAYA-RODRIGUEZ, M. T.; LEONARDI, S. M. Avaliação sedimentológica de uma área carbonífera. In: CENTRO DE ECOLOGIA DA UNIVERSIDADE FEDERAL DO RIO GRANDE DO SUL (Org.). Carvão e meio ambiente. Porto Alegre: Universidade Federal do Rio Grande do Sul, 2000. p.554-595

MOTA-MARQUES, D. M. L.; SUMINSKY, M.; BRUM, C. P. Ecossistemas criados (banhados): importância dos macrófitos no controle de $\mathrm{pH}$ de drenagem ácida In: CENTRO DE ECOLOGIA DA UNIVERSIDADE FEDERAL DO RIO GRANDE DO SUL (Org.). Carvão e meio ambiente. Porto Alegre: Universidade Federal do Rio Grande do Sul, 2000. p.743-748.

POMPÊO, M. L. M. et al. Qualidade da água em região alterada pela mineração de carvão na microbacia do rio Fiorita (Siderópolis, Estado de Santa Catarina, Brasil). Acta

Scientiarum. Biological Sciences, v.26, n.2, p.125-136, 2004.

R. Árvore, Viçosa-MG, v.34, n.1, p.177-188, 2010
PROCHNOW, T. R.; PORTO, M. L. Avaliação de uma área de rejeitos da mineração de carvão com vistas à bioindicadores vegetais para metais pesados. In: CENTRO DE ECOLOGIA DA UNIVERSIDADE FEDERAL DO RIO GRANDE DO SUL (Org.). Carvão e meio ambiente. Porto Alegre: Universidade Federal do Rio Grande do Sul, 2000. p.673-694.

QUARITI, O. et al. Cadmium and copper-induced changes in tomato membrane lipids.

Phytochemistry, v.45, n.7, p.1343-1350, 1997.

REITZ, R. Tifáceas. Flora ilustrada catarinense. Itajaí: Herbário Barbosa Rodrigues, 1984. 16p.

SKOUSEN, J. et al. Acid mine drainage treatmente with wetlands and anoxic limestone drains. In: KENT, D. M. (Ed.). Applied wetlands science and technology. Boca Raton: Lewis Publication, 1994. p.263-281.

SMITH, L. A. et al. Remedial options for metals-contaminated soil. New York: CRC Press, 1995. 453p.

SOARES, C. R. F. S. et al. Fitotoxidez de cádmio para Eucalyptus maculata e E. urophylla em solução nutritiva. Revista Árvore, v.29, n.2, p.175-183, 2005.

SOARES, C. R. F. S. et al. Acúmulo e distribuição de metais pesados nas raízes, caule e folhas de mudas de árvores em solo contaminado por rejeitos de indústria de zinco. Revista Brasileira Fisiologia Vegetal, v.13, n.3, p.302-315, 2001.

TAYLOR, G. J.; CROWDER, A. A. Uptake and accumulation of copper, nickel, and iron by Typha latifolia in wetlands of the Sudbury, Ontario region. Canadian Journal Botany, v.61, n.7, p.63-73, 1983.

VARDANYAN, L. G.; INGOLE, B. S. Studies on heavy metal accumulation in aquatic macrophytes from Sevan (Armênia) and Carambolin (Índia) lake systems.

Environment International, v.32, n.2, p.208-218, 2006. 
YE, Z. H. et al. Trace E element removal from coal ash leachate by a 10-year-old constructed wetland. Journal Environmental Quality, v.30, n. 5, p.1710-1719, 2001a.

YE, Z. H. et al. Removal and distribution of $\mathrm{Fe}, \mathrm{Mn}, \mathrm{Co}$ e Ni within a Pennsylvania constructed wetlands treating, coal combustion by-product leachate. Journal Environmental Quality, v.30, n. 5, p.1463-1473, 2001b.

ZANARDI Jr., V.; PORTO, M.L. Avaliação do sistema de lagoas em áreas de mineração de carvão a céu aberto: metais pesados em água, planta e substrato. Boletim do Instituto de Biociências, n.49, p.1-83, 1991.

ZAR, J. Bioestatiscal analisis. New Jersey: Prentice-Hall, 1985. 656p.
ZOCCHE-DE-SOUZA, P.; COSTA, S.; ZOCCHE, J. J. Baccharis trimera Less. DC. como indicadora da recuperação de Áreas mineradas de carvão.

Revista Brasileira de Biociências, v.5, Suplemento 1, p.702-704, 2007.

ZOCCHE, J. J. Comunidades vegetais de savana sobre estruturas mineralizadas de cobre na Mina Volta Grande, Lavras do Sul, RS. 2002. 205f. Tese (Doutorado em Ciências) -Universidade Federal do Rio Grande do Sul, Porto Alegre, 2002.

ZOCCHE, J. J. Metais pesados (Fe, Mn e Zn) no solo construído e na vegetação das antigas bacias de decantação do lavador de Capivari, Capivari de Baixo, SC. In: SIMPÓSIO NACIONAL E CONGRESSO LATINO-AMERICANODE RECUPERAÇÃODE ÁREAS DEGRADADAS, 6., 2005, Curitiba. Anais... Curitiba: SOBRADE, 2005.p.117-124. 
